\title{
Observables and initial conditions for self-similar ellipsoidal flows
}

\author{
T. Csörgö ${ }^{1,2}$, S.V. Akkelin ${ }^{3}$, Y. Hama ${ }^{2}$, B. Lukács ${ }^{1}$ and Yu.M. Sinyukov ${ }^{2,3}$ \\ ${ }^{1}$ MTA KFKI RMKI, H-1525 Budapest 114, POB 49, Hungary \\ ${ }^{2}$ IF-USP, C.P. 66318, 05389-970 São Paulo, SP, Brazil, \\ 3 Bogolyubov Institute for Theoretical Physics, Kiev 03143, Metrologichna 14b, Ukraine
}

\begin{abstract}
Single-particle spectra and two-particle Bose-Einstein correlation functions are determined analytically utilizing a self-similar solution of non-relativistic hydrodynamics for ellipsoidally-symmetric, expanding fireballs, by assuming that the symmetry axes of the ellipsoids are tilted in the frame of the observation. The directed, elliptic and third flows are calculated analytically. The mass dependences of the slope parameters in the principal directions of the expansion, together with the mass and angular dependences of the HBT radius parameters, reflect directly the ellipsoidal properties of the flow.
\end{abstract}

Introduction - The equations of hydrodynamics reflect the local conservation of matter, momentum and energy. These equations are well suited to study problems related to flows in various fields ranging from evolution of galaxies in astrophysics to heavy-ion and elementaryparticle collisions in high-energy physics. The finding of exact self-similar hydro solutions sometimes represents essential progress in physics, as the discovery of the Hubble flow of our Universe or the Bjorken flow of ultrarelativistic heavy-ion collisions.

In this Letter we consider the case of a non-relativistic hydrodynamical problem with ellipsoidal symmetry. Our goal is to demonstrate the influence of initial conditions on the final state observables, utilizing an explicit, exact and simple analytic solution of fireball hydrodynamics. In particular, we attempt to understand the relationship between the initial conditions (the ellipsoidal asymmetry and the tilt of the major axis) and the final observables.

The new family of self-similar ellipsoidal solutions A self-similar solution of non-relativistic hydrodynamics with ideal gas equation of state and a generalized (direction-dependent) Hubble flow, a three-dimensional ellipsoidal Gaussian density profile and a homogeneous, space-indepenent temperature profile has just been found in ref. [1]. This solution has many interesting properties, e.g. the partial differential equations of hydrodynamics are reduced to ordinary differential equations corresponding to a Hamiltonian motion of a massive particle in a non-central repulsive potential. These results correspond to the generalization of earlier, data motivated hydrodynamical parameterizations and/or solutions of refs. [2-8] to ellipsoidal symmetry and non-central heavy-ion collisions with homogeneous temperature profile.

Below we generalize the hydrodynamic solution of ref. [1] for some fairly wide family of thermodynamically consistent equations of state, and calculate analytically all the observables of non-central collisions. It allows, in principle, to solve an inverse problem, namely, given an (in general non-ideal) equation of state, to restore the initial conditions from the observables. However, we do not aim here to apply directly the new hydro solution to data fitting in high-energy heavy-ion physics. In order to reach the level of data fitting, generalizations to relativistic flow patterns, more realistic equations of state and temperature profiles are needed. Some of these generalizations seem to be straightforward and are in progress $[9,10]$.

Consider the non-relativistic hydrodynamical problem, as given by the continuity, Euler and energy equations:

$$
\begin{aligned}
\partial_{t} n+\nabla \cdot(n \mathbf{v}) & =0, \\
\partial_{t} \mathbf{v}+(\mathbf{v} \cdot \nabla) \mathbf{v} & =-(\nabla p) /(m n), \\
\partial_{t} \epsilon+\nabla \cdot(\epsilon \mathbf{v}) & =-p \nabla \cdot \mathbf{v},
\end{aligned}
$$

where $n$ denotes the particle number density, $\mathbf{v}$ stands for the non-relativistic (NR) flow velocity field, $\epsilon$ for the NR energy density, $p$ for the pressure and in the following the temperature field is denoted by $T$. This set of equations are closed by some equation of state (EoS). We have chosen analytically solvable generalization of NR ideal gas equations of state:

$$
p=n T, \quad \epsilon=\kappa(T) n T,
$$

which allow to study the solutions of NR hydro equations for any temperature dependent ratio of pressure to energy density, $p / \epsilon=1 / \kappa(T)$. The EoS (4) are thermodynamically consistent for any function $\kappa(T)$, as can be checked by using the free energy density $f(T, n)$ and the relations

$$
p=n \frac{\partial f}{\partial n}-f, \quad \epsilon=f-T \frac{\partial f}{\partial T} .
$$

The function $\kappa(T)$ characterizes the $p / \epsilon$ ratio for a broad variety of materials: e.g. a non-relativistic ideal gas yields $\kappa(T)=3 / 2$. Note, that for finite-size systems phase transition can occupy certain temperature interval similar to a crossover. Then one can model such a change of the pressure to energy density ratio at phase transition from deconfined quark matter to hadronic one by means of smooth variations of the values of $\kappa(T)$ in certain temperature domain. Note also that it is usual to introduce the speed of sound as $c_{s}^{2}=d p / d \epsilon=1 / \kappa(T)$, so we model the change in the equation of state essentially with the help of a temperature dependent speed of sound.

For reasons of convenience we choose $n, \mathbf{v}$ and $T$ as the independent hydrodynamic variables. The NR hydro equations are solved, similarly as it was done for the 
case of NR ideal gas EoS in ref. [1], by the following selfsimilar, ellipsoidally symmetric density and flow profiles:

$$
\begin{aligned}
n\left(t, \mathbf{r}^{\prime}\right) & =n_{0} \frac{V_{0}}{V} \exp \left(-\frac{\mathrm{r}_{\mathrm{x}}^{\prime 2}}{2 \mathrm{X}^{2}}-\frac{\mathrm{r}_{\mathrm{y}}^{\prime 2}}{2 \mathrm{Y}^{2}}-\frac{\mathrm{r}_{\mathrm{z}}^{\prime 2}}{2 \mathrm{Z}^{2}}\right), \\
\mathbf{v}^{\prime}\left(t, \mathbf{r}^{\prime}\right) & =\left(\frac{\dot{X}}{X} r_{x}^{\prime}, \frac{\dot{Y}}{Y} r_{y}^{\prime}, \frac{\dot{Z}}{Z} r_{z}^{\prime}\right),
\end{aligned}
$$

where the variables are defined in a center of mass frame $K^{\prime}$, but with the axes pointing to the principal directions of the expansion. The time dependent scale parameters are denoted by $(X, Y, Z)=(X(t), Y(t), Z(t))$, the typical volume of the expanding system is $V=X Y Z$, and the initial temperature and volume are $T_{0}=T\left(t_{0}\right)$ and $V_{0}=V\left(t_{0}\right)$, and $n_{0}$ is a constant. The time evolution of the radius parameters $X, Y, Z$ and temperature $T$ are governed by the ordinary differential equations

$$
\begin{array}{r}
\ddot{X} X=\ddot{Y} Y=\ddot{Z} Z=\frac{T}{m}, \\
\dot{T} \frac{d}{d T}(\kappa T)+T\left(\frac{\dot{X}}{X}+\frac{\dot{Y}}{Y}+\frac{\dot{Z}}{Z}\right)=0 .
\end{array}
$$

Note that the equation for the time dependence of the temperature can be integrated in a straigthforward manner to find

$$
\frac{V_{0}}{V}=\exp \left[\kappa(T)-\kappa\left(T_{0}\right)\right] \exp \int_{T_{0}}^{T} \frac{d T^{\prime}}{T^{\prime}} \kappa\left(T^{\prime}\right),
$$

and this equation further simplified, in the case of a temperature independent $\kappa$, as

$$
T=T_{0}\left(\frac{V_{0}}{V}\right)^{1 / \kappa} .
$$

Observables from the new solution - In order to evaluate the measurable quantities, any hydrodynamical solution has to be supplemented with an additional freeze-out criterion, that specifies the end of the hydrodynamical evolution. Here we assume sudden particle freeze-out at a constant temperature $T\left(t_{f}, \mathbf{r}\right)=T_{f}$ where EoS corresponds approximately to ideal gas $\left(\kappa\left(T_{f}\right)=3 / 2\right)$. This freeze-out condition is reached everywhere at the same time in the considered class of exact hydrodynamical solutions and it is motivated by the simplicity of the results. Then, the emission function is proportional to

$$
S\left(t, \mathbf{r}^{\prime}, \mathbf{k}^{\prime}\right) \propto \mathrm{e}^{-\frac{\left(x \mathbf{k}^{\prime}-m \mathbf{v}^{\prime}\right)^{2}}{2 m m T_{f}}-\frac{r_{x}^{\prime 2}}{2 X_{f}^{2}}-\frac{r_{y}^{\prime 2}}{2 Y_{f}^{2}}-\frac{r_{z}^{\prime}}{2 Z_{f}^{2}}} \delta\left(t-t_{f}\right) .
$$

Single particle spectrum - The single-particle spectrum and the two-particle correlation function can be evaluated similarly to that of ref. [3,4]:

$$
E \frac{d^{3} n}{d \mathbf{k}^{\prime}} \propto E \exp \left(-\frac{k_{x}^{\prime 2}}{2 m T_{x}^{\prime}}-\frac{k_{y}^{\prime 2}}{2 m T_{y}^{\prime}}-\frac{k_{z}^{\prime 2}}{2 m T_{z}^{\prime}}\right),
$$

$$
\begin{aligned}
& T_{x}^{\prime}=T_{f}+m \dot{X}_{f}^{2}, \\
& T_{y}^{\prime}=T_{f}+m \dot{Y}_{f}^{2}, \\
& T_{z}^{\prime}=T_{f}+m \dot{Z}_{f}^{2} .
\end{aligned}
$$

Here $E=m+\mathbf{k}^{\prime 2} /(2 m)$ in the non-relativistic limit we are considering, $\mathbf{k}^{\prime}=\left(k_{x}^{\prime}, k_{y}^{\prime}, k_{z}^{\prime}\right)$ stands for the momentum vector in $K^{\prime}, X_{f}=X\left(t_{f}\right)$, etc. In the spherically symmetric case of $X=Y=Z=R$, we recover the earlier results [3,4], with $\langle u\rangle=\dot{R}$ and $T_{\text {eff }}=T_{f}+m\langle u\rangle^{2}$.

The observables are determined in the center of mass frame of the collision, $K$, where the $r_{z}$ axis points to the direction of the beam and the $r_{x}$ axis to that of the impact parameter. In this frame, the coordinates and the momenta are denoted by $\mathbf{x}$ and $\mathbf{k}$. We assume that the initial state of the hydrodynamic evolution corresponds to a rotated ellipsoid in $K$. The tilt angle $\theta$ represents the rotation of the major (longitudinal) direction of expansion, $r_{z}^{\prime}$ from the beam axis $r_{z}$. Hence the event plane is the $\left(r_{x}^{\prime}, r_{z}^{\prime}\right)$ plane, which is the same as the $\left(r_{x}, r_{z}\right)$ plane. The (zenithal) angle between directions $r_{z}$ and $r_{z}^{\prime}$ is $\theta$, while the (azimuthal) angle between the transverse momentum $\mathbf{k}_{t}$ and the event plane is $\phi$.

The ellipsoidal spectrum of eq. (13) generates the following $\phi$ averaged single-particle spectrum in the $K$ frame:

$$
\begin{aligned}
\frac{d^{2} n}{2 \pi k_{t} d k_{t} d k_{z}} & \propto \exp \left(-\frac{k_{t}^{2}}{2 m T_{\mathrm{eff}}}-\frac{k_{z}^{2}}{2 m T_{z}}\right) f(v, w), \\
\frac{1}{T_{z}} & =\frac{\cos ^{2} \theta}{T_{z}^{\prime}}+\frac{\sin ^{2} \theta}{T_{x}^{\prime}} \\
\frac{1}{T_{x}} & =\frac{\cos ^{2} \theta}{T_{x}^{\prime}}+\frac{\sin ^{2} \theta}{T_{z}^{\prime}} \\
\frac{1}{T_{\mathrm{eff}}} & =\frac{1}{2}\left(\frac{1}{T_{x}}+\frac{1}{T_{y}^{\prime}}\right) \\
w & =\frac{k_{t}^{2}}{4 m}\left(\frac{1}{T_{y}^{\prime}}-\frac{1}{T_{x}}\right) \\
v & =-\frac{k_{t} k_{z}}{2 m} \sin (2 \theta)\left(\frac{1}{T_{x}^{\prime}}-\frac{1}{T_{z}^{\prime}}\right), \\
f(v, w) & \approx I_{0}(w)+\frac{v^{2}}{4}\left[I_{0}(w)+I_{1}(w)\right],
\end{aligned}
$$

where $f(v, w)$ is calculated for $|v| \ll 1$ and $I_{n}(w)=$ $\frac{1}{\pi} \int_{0}^{\pi} d z \cos (n z) \exp [w \cos (z)]$ is the modified Bessel function of order $n(n=0,1, \ldots)$. For small ellipsoidal asymmetries, $w \ll 1, I_{0}(w) \simeq 1$ and the effective temperature parameter in the transverse direction is the harmonic mean of the temperature parameters of the principal directions of expansion (projected to the transverse plane). As $T_{z}^{\prime} \geq T_{x}^{\prime} \geq T_{y}^{\prime}$ is expected from the initial conditions, we obtain $T_{x} \geq T_{x}^{\prime}$ and $T_{z} \leq T_{z}^{\prime}$.

The flow coefficients $v_{n}$ are defined as

$$
\frac{d^{3} n}{d k_{z} k_{t} d k_{t} d \phi}=\frac{d^{2} n}{2 \pi d k_{z} k_{t} d k_{t}}\left[1+2 \sum_{n=1}^{\infty} v_{n} \cos (n \phi)\right] \text {. }
$$


Here $v_{1}$ is called the directed flow, $v_{2}$ the elliptic flow and $v_{3}$ the third flow. The transverse- and longitudinalmomentum dependence of the $v_{n}$ flow components can be written in terms of $v$ and $w$. Assuming that the tilt angle $\theta$ or the anisotropy is small, $|v| \ll 1$, the directed, elliptic and third flow components are evaluated as

$$
\begin{aligned}
& v_{1}=\frac{v}{2}\left[1+\frac{I_{1}(w)}{I_{0}(w)}\right], \\
& v_{2}=\frac{I_{1}(w)}{I_{0}(w)}+\frac{v^{2}}{8}\left[1+\frac{I_{2}(w)}{I_{0}(w)}-2\left(\frac{I_{1}(w)}{I_{0}(w)}\right)^{2}\right], \\
& v_{3}=\frac{v}{2} \frac{I_{2}(w)+I_{1}(w)}{I_{0}(w)} .
\end{aligned}
$$

An angular tilt $\theta \neq 0$ is evidenced by the rise of the directed and third flows as a function of rapidity $y=$ $0.5 \ln \left[\left(E+k_{z}\right) /\left(E-k_{z}\right)\right]$ and by a minimum of the elliptic flow at mid-rapidity, see Fig. 1. This and other features are in qualitative agreement with most of the data on intermediate- and high-energy heavy-ion collisions [11-14], suggesting that in non-central collisions the dominant longitudinal direction of expansion is slightly deviating from the beam direction. A more straightforward proof of the ellipsoidal nature of the flow can be obtained by determining the mass dependence of the parameters $T_{x}^{\prime}, T_{y}^{\prime}$ and $T_{z}^{\prime}$, cf. eqs. (14-16) and Fig. 2.

Two-particle correlations - The two-particle BoseEinstein correlation function (BECF) is related to a Fourier-transform of the emission (or source) function $S\left(t, \mathbf{r}^{\prime}, \mathbf{k}^{\prime}\right)$ of eq. (12), see e.g. refs. [3,4,6]. If the corehalo picture [15] is valid, an effective intercept parameter $\lambda \equiv \lambda(\mathbf{k})=\left[N_{c}(\mathbf{k}) / N(\mathbf{k})\right]^{2}$ appears, that measures the fraction of particles emitted directly from the core. The two-particle BECF is diagonal in $K^{\prime}$, as

$$
\begin{aligned}
C\left(\mathbf{K}^{\prime}, \mathbf{q}^{\prime}\right) & =1+\lambda \exp \left(-q_{x}^{\prime 2} R_{x}^{\prime 2}-q_{y}^{\prime 2} R_{y}^{\prime 2}-q_{z}^{\prime 2} R_{z}^{\prime 2}\right), \\
\mathbf{K}^{\prime} & =\mathbf{K}_{12}^{\prime}=0.5\left(\mathbf{k}_{1}^{\prime}+\mathbf{k}_{2}^{\prime}\right), \\
\mathbf{q}^{\prime} & =\mathbf{q}_{12}^{\prime}=\mathbf{k}_{1}^{\prime}-\mathbf{k}_{2}^{\prime}=\left(q_{x}^{\prime}, q_{y}^{\prime}, q_{z}^{\prime}\right), \\
R_{x}^{\prime-2} & =X_{f}^{-2}\left(1+\frac{m}{T_{f}} \dot{X}_{f}^{2}\right), \\
R_{y}^{\prime-2} & =Y_{f}^{-2}\left(1+\frac{m}{T_{f}} \dot{Y}_{f}^{2}\right), \\
R_{z}^{\prime-2} & =Z_{f}^{-2}\left(1+\frac{m}{T_{f}} \dot{Z}_{f}^{2}\right) .
\end{aligned}
$$

These radius parameters measure the lengths of homogeneity [16]. They are dominated by the shortest of the geometrical scales $\left(X_{f}, Y_{f}, Z_{f}\right)$ and the corresponding thermal scales defined by $\left(X_{T}, Y_{T}, Z_{T}\right)=$ $\sqrt{\frac{T_{f}}{m}}\left(\frac{X_{f}}{X_{f}}, \frac{Z_{f}}{Z_{f}}, \frac{Z_{f}}{Z_{f}}\right)$, generalizing the results of refs. [3,6,7] to ellipsoidal flows. The geometrical scales characterize the spatial variation of the fugacity term $\mu\left(t, \mathbf{r}^{\prime}\right) / T\left(t, \mathbf{r}^{\prime}\right)$, while the thermal scales characterize the spatial variations of the Boltzmann term $E_{\mathrm{loc}}^{\prime}\left(t, \mathbf{r}^{\prime}\right) / T\left(t, \mathbf{r}^{\prime}\right)$, both of them evaluated at the point of maximal emittivity. In the $K^{\prime}$ frame, cross-terms [17] vanish, $R_{i \neq j}^{2}=0$, if the emission is sudden.

If the particle emission is gradual, but it happens in a narrow interval $\Delta t$ centered at $t_{f}$, then the BECF can be evaluated using the replacement $\delta\left(t-t_{f}\right) \rightarrow$ $\left(2 \pi \Delta t^{2}\right)^{-1 / 2} \exp \left[-\left(t-t_{f}\right)^{2} / 2 \Delta t^{2}\right]$ in eq. (12), if $\Delta t \dot{X}_{f}<<$ $X_{f}$, etc. Hence all the previous radius components, including the cross-terms, are extended with an additional term $\delta R_{i j}^{\prime 2}=\beta_{i}^{\prime} \beta_{j}^{\prime} \Delta t^{2}$, where $\boldsymbol{\beta}^{\prime}=\left(\mathbf{k}_{1}^{\prime}+\mathbf{k}_{2}^{\prime}\right) /\left(E_{1}^{\prime}+E_{2}^{\prime}\right)$ is the velocity of the pair in $K^{\prime}$.

The BECF's are usually given in the side-outlongitudinal or Bertsch-Pratt (BP) parameterization. The longitudinal direction, $r_{\text {long }} \equiv r_{1}$ in $\mathrm{BP}$ coincides with the beam direction. The plane orthogonal to the beam is decomposed to a direction parallel to the mean transverse momentum of the pair, $r_{\text {out }} \equiv r_{\mathrm{o}}$, and the one perpendicular both to this and the beam direction, $r_{\text {side }}=r_{\mathrm{s}}$. The mean velocity of the particle pair can be written in $\mathrm{BP}$ as $\boldsymbol{\beta}=\left(\beta_{\mathrm{o}}, 0, \beta_{1}\right)$, where $\beta_{\mathrm{o}}=\beta_{t}$. Let $\phi$ denote the angle of the event plane and the mean transverse momentum of the measured pair. The result is

$$
\begin{aligned}
C_{2}(\mathbf{K}, \mathbf{q}) & =1+\lambda \exp \left(-\sum_{i, j=\mathrm{s}, \mathrm{o}, 1} q_{i} q_{j} R_{i j}^{2}\right), \\
R_{\mathrm{s}}^{2} & =R_{y}^{\prime 2} \cos ^{2} \phi+R_{x}^{2} \sin ^{2} \phi, \\
R_{\mathrm{o}}^{2} & =R_{x}^{2} \cos ^{2} \phi+R_{y}^{\prime 2} \sin ^{2} \phi+\beta_{t}^{2} \Delta t^{2}, \\
R_{1}^{2} & =R_{z}^{\prime 2} \cos ^{2} \theta+R_{x}^{\prime 2} \sin ^{2} \theta+\beta_{1}^{2} \Delta t^{2}, \\
R_{\mathrm{ol}}^{2} & =\left(R_{x}^{\prime 2}-R_{z}^{\prime 2}\right) \cos \theta \sin \theta \cos \phi+\beta_{t} \beta_{1} \Delta t^{2}, \\
R_{\mathrm{os}}^{2} & =\left(R_{x}^{2}-R_{y}^{\prime 2}\right) \cos \phi \sin \phi, \\
R_{\mathrm{sl}}^{2} & =\left(R_{x}^{\prime 2}-R_{z}^{\prime 2}\right) \cos \theta \sin \theta \sin \phi,
\end{aligned}
$$

where an auxiliary quantity is introduced as

$$
R_{x}^{2}=R_{x}^{\prime 2} \cos ^{2} \theta+R_{z}^{\prime 2} \sin ^{2} \theta .
$$

These results imply that all the radius parameters oscillate in the $K$ frame. In particular, a $\phi$ dependent oscillation appears in the radius parameters indexed either by the side or the out direction, as illustrated in Fig. 3 . These oscillations are similar to those obtained in ref. [18], corresponding to $\theta=0$. We find that the radius parameters indexed by the longitudinal direction depend also on the zenithal angle $\theta$. A toy model for tilted $(\theta \neq 0)$ ellipsoidal static pion sources was introduced in refs. [19], to understand the $\phi$ dependent oscillations of measured HBT radii at AGS energies. In our case, the amplitude of the oscillations is reduced for heavier particles due to the hydrodynamic expansion, which results in a decrease of the lengths of homogeneity with increasing mass. The oscillations of the radius parameters were not related before either to hydrodynamic flow with ellipsoidal symmetry and tilt of the major axis or connected to the initial conditions of a hydrodynamic expansion.

A check of the applicability of our hydrodynamic solution is that the BECF and the single particle spectrum 
become diagonal (after removing a term of $\beta_{i} \beta_{j} \Delta t^{2}$ from all the HBT radius parameters) in the same frame, see eqs. $(13,14-16)$ and eqs. $(28,31-33)$. This frame is $K^{\prime}$, the natural System of Ellipsoidal Expansion or SEE.

Summary - We have analytically evaluated the singleparticle spectrum and the two-particle Bose-Einstein correlation function for a self-similarly expanding, exact, ellipsoidal solution of the non-relativistic hydrodynamical equations, assuming a constant freeze-out temperature.

The parameters of the hydro solution at freeze-out time $T_{f},\left(X_{f}, Y_{f}, Z_{f}\right)$ and $\left(\dot{X}_{f}, \dot{Y}_{f}, \dot{Z}_{f}\right)$, can be reconstructed from the measurement of the single particle spectrum and the two-particle correlation functions. The direction of the major axis of expansion in the center of mass frame of the collision is characterized by the polar angles $(\theta, 0)$. With the exception of $R_{1}$, all the radius parameters oscillate as function of $\phi$, while the radius parameters $R_{1}^{2}$, $R_{\mathrm{ol}}^{2}, R_{\mathrm{sl}}^{2}$ depend also on $\theta$. All the radius parameters decrease with increasing mass, including all the cross terms. If $\theta \neq 0$, the effective temperature in the transverse direction is increased by a contribution from the longitudinal expansion.

The initially more compressed longitudinal and impact parameter directions (the $r_{z}$ and $r_{x}$ directions) expand more dynamically [1,9], that implies $T_{z}^{\prime} \geq T_{x}^{\prime} \geq T_{y}^{\prime}$. The initial time $t_{0}$ can be identified from the requirement that $\dot{Y}\left(t_{0}\right)=0$. The initial conditions for this hydrodynamical system can be uniquely reconstructed from final state measurements. The function $\kappa(T)$ in the EoS influences only the time evolution of the scales $(X, Y, Z)$ and temperature $T$. So, for a given EoS, one can uniquely reconstruct the initial conditions of hydrodynamic evolution from final ones.

We have deliberately chosen the presentation as simple as possible, which limits the direct applicability of our results in high-energy heavy-ion collisions only to sufficiently small transverse momentum, $p_{t} \ll m$, at midrapidity. But the scheme permits generalization in many points and still the qualitative features of our results may survive even in the relativistic regime. Generalizations to some relativistic flows have been described in ref. [10]. Ref. [9] includes another extension to an arbitrary, inhomogeneous, ellipsoidally symmetric initial temperature profile, which does not change the time evolution of the scale parameters. It turns out that each of these generalizations is essentially straightforward.

Although we considered a non-relativisitic problem, our results provide generic insight into the time evolution of non-central heavy-ion collisions and relate initial conditions to final-state observables in a simple and straightforward manner impossible before.

Acknowledgments: This work has been supported by the grants FAPESP 00/04422-7, 99/09113-3, 01/05122-0 of São Paulo, Brazil, by the Hungarian - Ukrainian S\&T grant 45014 (2M/125-199), the OTKA grant T026435 and the NWO - OTKA grant N 25487. Y. S. and S. A. have been supported by CNRS and the DLR grant 2M-141- 2000.
[1] S.V. Akkelin, T. Csörgő, B. Lukács, Yu. M. Sinyukov and M. Weiner, Phys. Lett. B505 (2001) 64.

[2] J. Bondorf, S. Garpman and J. Zimányi, Nucl. Phys. A296 (1978) 320.

[3] T. Csörgő, B. Lörstad and J. Zimányi; Phys. Lett. B338 (1994) 134; nucl-th/9408022.

[4] P. Csizmadia, T. Csörgő and B. Lukács, Phys. Lett. B443 (1998) 21; nucl-th/9805006.

[5] T. Csörgő, nucl-th/9809011.

[6] J. Helgesson, T. Csörgö, M. Asakawa and B. Lörstad, Phys. Rev. C56 (1997) 2626.

[7] T. Csörgő and B. Lörstad, Phys. Rev. C54 (1996) 1390; T. Csörgő and B. Lörstad, Nucl. Phys. A590 (1995) 465c.

[8] J.N. De, S.I.A. Garpman, D. Sperber, J.P. Bondorf and J. Zimányi, Nucl. Phys. A305 (1978) 226.

[9] T. Csörgő, hep-ph/0111139.

[10] T. Csörgő, F. Grassi, Y. Hama and T. Kodama, hepph/0203204; hep-ph/0204300.

[11] P. Danielewicz, Nucl. Phys. A685 (2001) 368, and references therein.

[12] J. Bächler et al, Nucl. Phys. A661 (1999) 341c.

[13] K.H. Ackermann et al, Phys. Rev. Lett. 86 (2001) 402.

[14] L.P. Csernai and D. Röhrich, Phys. Lett. B458 (1999) 454; nucl-th/9908034.

[15] T. Csörgő, B. Lörstad and J. Zimányi, Z. Phys. C71 (1996) 491; hep-ph/9411307.

[16] Yu. M. Sinyukov, Nucl. Phys. A566 (1994) 589c .

[17] S. Chapman, P. Scotto, U. Heinz, Phys. Rev. Lett. 74 (1995) 4400.

[18] U.A. Wiedemann, Phys. Rev. C57 (1998) 266; H. Heiselberg and A. Levy, Phys. Rev. C59 (1999) 2716.

[19] M.A. Lisa, U. Heinz and U.A. Wiedemann, Phys. Lett. B489 (2000) 287; nucl-th/0003022; M. A. Lisa et al, E895 Collaboration, Nucl. Phys. A661 (1999) 444c.

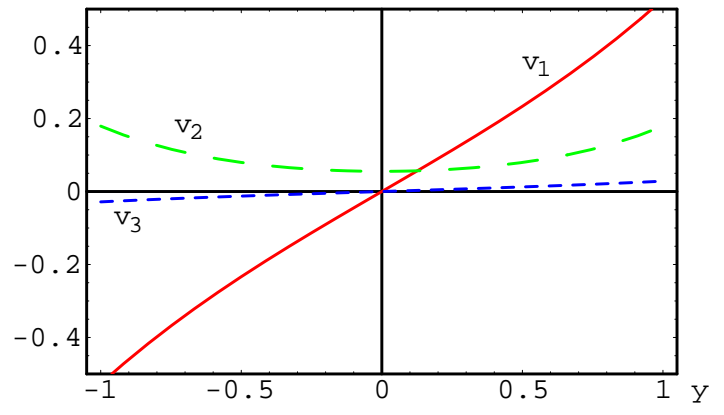

FIG. 1. The directed, elliptic and third flows $v_{1}, v_{2}$, $v_{3}$ are illustrated, respectively with solid, long-dashed and short-dashed lines, as a function of rapidity, for $m=940$ $\mathrm{MeV}, T_{x}^{\prime}=200 \mathrm{MeV}, T_{y}^{\prime}=150 \mathrm{MeV}, T_{z}^{\prime}=700 \mathrm{MeV}$, at a fixed $k_{t}=500 \mathrm{MeV}$ and $\theta=\pi / 5$, see eqs. (25-27). 


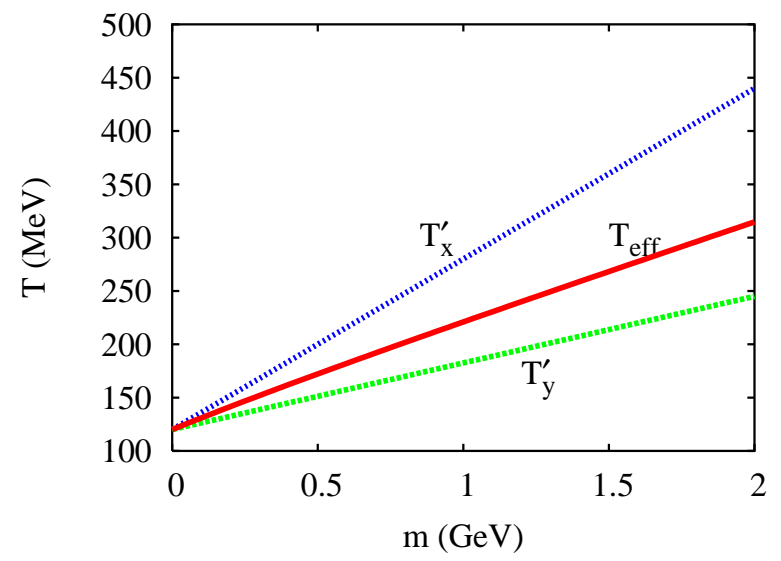

FIG. 2. The linear mass dependence of the effective temperatures in the transverse directions, $T_{x}^{\prime}, T_{y}^{\prime}$ and their (harmonic) average $T_{\text {eff }}$ for non-central heavy-ion collisions, if $T_{f}=120 \mathrm{MeV}, \dot{X}=0.4$, and $\dot{Y}=0.25$ and $\theta=0$.

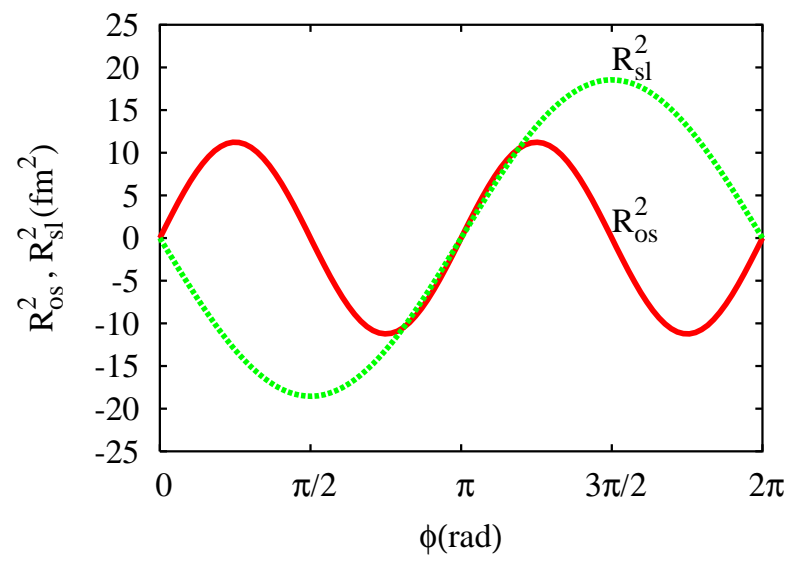

FIG. 3. The out-side and the side-long cross terms are plotted as a function of the polar angle $\phi$, for $R_{x}^{\prime}=5 \mathrm{fm}, R_{y}^{\prime}=4$ $\mathrm{fm}, R_{z}^{\prime}=8 \mathrm{fm}$ and $\theta=\pi / 5$. Note that $R_{\mathrm{sl}}^{2} \propto \sin (\phi)$ while $R_{\mathrm{os}}^{2} \propto \sin (2 \phi)$. See eqs. (39) and (40). 